\title{
Médiévales
}

Langues, Textes, Histoire

76 | printemps 2019

Du nouveau en archives

\section{Comptabilité publique et innovation à la fin du Moyen Âge : les institutions princières et l'émergence d'une culture numérique dans un ordre conservateur}

Public accounting and innovation in the Late Middle Ages: Princely institutions and the growth of numerical culture in a conservative order

Jean-Baptiste Santamaria

\section{(2) OpenEdition}

Journals

Édition électronique

URL : https://journals.openedition.org/medievales/9974

DOI : 10.4000/medievales.9974

ISSN : 1777-5892

Éditeur

Presses universitaires de Vincennes

Édition imprimée

Date de publication : 5 septembre 2019

Pagination : 113-132

ISBN : 978-2-37924-028-7

ISSN : 0751-2708

Référence électronique

Jean-Baptiste Santamaria, «Comptabilité publique et innovation à la fin du Moyen Âge : les institutions princières et l'émergence d'une culture numérique dans un ordre conservateur »,

Médiévales [En ligne], 76 | printemps 2019, mis en ligne le 01 janvier 2021, consulté le 22 avril 2022

URL : http://journals.openedition.org/medievales/9974; DOI : https://doi.org/10.4000/medievales. 9974 


\author{
Comptabilité publique et innovation \\ à la fin du Moyen Âge \\ Les institutions princières et l'émergence \\ d'une culture numérique dans un ordre conservateur
}

«Faut-il dire cette chose honteuse que notre Chambre des comptes attendit au dix-septième siècle pour adopter les chiffres arabes, sans lesquels on ne peut faire le plus simple calcul ${ }^{1}$ ? » Si l'amalgame fait par Jules Michelet entre institution de contrôle et archaïsme ${ }^{2}$ a encore pu être accepté par Lucien Febvre, pour qui le calcul était assez secondaire dans la formation des gens des comptes ${ }^{3}$, le lien est désormais bien établi entre «l'essor des institutions et l'amélioration des techniques administratives », selon les mots de Jean-Philippe Genet ${ }^{4}$. La relation entre innovation institutionnelle et documentaire peut cependant s'avérer ambivalente dans une période où la novelleté fait figure d'épouvantail politique, et où l'innovation financière n'est guère revendiquée comme telle ${ }^{5}$, mais promeut largement la compétence la plus pointue, faisant du maître des comptes un « expers et cognoissans en fait de compte ${ }^{6} »$ : les rapports des experts à l'innovation

1. J. MichelET, Histoire de France, Paris, (1854) 1855, vol. 7, p. CL.

2. Un passage qui témoigne du lien très caricatural établi par l'auteur entre christianisme et archaïsme puisqu'il ajoute « Où paraissent les chrétiens, paraît le désert »...

3. L. FeBVRE, «Comptabilité et Chambre des Comptes », Annales d'histoire économique et sociale, 6 (1934), p. 148-153.

4. J.-P. GENET, "Conclusion [Les Chambres des comptes en France aux XIV et $\mathrm{XV}^{\mathrm{e}}$ siècles] », dans P. CONTAMINE et O. MATTÉONI éd., La France des principautés : les Chambres des comptes, XIVe et XVe siècles, Paris, 1996, p. 267-279.

5. P. CONTAMINE, « La crise de la royauté française au XIV $V^{e}$ siècle : réformation et innovation dans le Songe du Vieil Pelerin (1389) de Philippe de Mézières », dans Tradition, Innovation, Invention. Fortschrittsverweigerung und Fortschrittsbewußtsein, Berlin, 2005, p. 361-379 ; L. SCORDIA, "Le roi doit vivre du sien » : la théorie de l'impôt en France, XIII ${ }^{\text {- }}$ $X V^{e}$ siècles, Paris, 2005.

6. J.-B. SANTAMARIA, «"Expers et cognoissans en fait de comptes". Conseil et expertise comptable dans les Pays-Bas bourguignons, fin XIV -début XV siècle », dans Expertise et conseil au Moyen Âge. Consilium quaeritur a perito, Actes du XLII ${ }^{\circ}$ congrès de la SHMESP, Paris, 2012, p. 201-213. 
peuvent être ambigus, allant de l'utilisation de la nouveauté pour dominer à la défense des usages dont ils sont les maîtres. On peut donc s'interroger sur la nature du rapport entre innovation documentaire comptable et processus d'institutionnalisation de l'administration. Par innovation, on entendra la manière dont une réalisation technique nouvelle s'impose dans les usages, phénomène qui peut s'avérer lent et relève souvent bien plus de l'imitation d'usages d'un domaine ou d'un lieu à l'autre que de véritables inventions ; par institutionnalisation, on désignera l'action de transférer les fonctions exercées par des individus à une organisation collective, légitimée, normée par des règles, un processus qui connaît un pic avec la création des Chambres des comptes, en particulier au XIV e siècle. Les effets de cette institutionnalisation sur la documentation nous intéresseront d'ailleurs autant que les effets de l'évolution documentaire sur l'apparition de telles structures. L'analyse partira d'un constat : nombre de changements documentaires se sont faits en amont de ce basculement. Nous en viendrons ensuite aux effets de la mise en place de ces institutions, à leur rôle dans la diffusion de nouvelles pratiques, et enfin à leur attitude parfois hostile à l'innovation.

\section{Un effort d'innovation documentaire souvent ancien}

Bien avant l'essor des Chambres des comptes, c'est au XII ${ }^{\mathrm{e}}$ siècle que la documentation comptable princière émerge, accompagnée de premières institutions : chambre apostolique romaine ${ }^{7}$; chambre des Renenghes flamande pour laquelle est rédigé le « gros brief » de Flandre ${ }^{8}$; échiquier et Pipe rolls anglais ${ }^{9}$. Les réformes de Philippe Auguste pour assurer le contrôle financier précèdent d'ailleurs de peu les premiers comptes conservés (1202$1203)^{10}$. Une fois installées dans le paysage, ces institutions enracinent des pratiques documentaires qui se trouvent fossilisées, et peuvent devenir un obstacle à l'innovation. Face aux changements des modes de gestion et de nature des revenus, le « gros brief » de Flandre devient un document inadapté à la réalité financière de la Flandre de la seconde moitié du XIII ${ }^{\mathrm{e}}$ siècle.

7. A. PARAVICINI BAgLiAni, La Cour des papes au XIII siècle, Paris, 1995 ; A. JAMME, «De la banque à la Chambre ? Naissance et mutations d'une culture comptable dans les provinces papales entre XIII ${ }^{\mathrm{e}}$ et $\mathrm{XV}^{\mathrm{e}}$ siècle », dans Offices, Écrit et Papauté (XIII ${ }^{e}-\mathrm{XVII}{ }^{e}$ siècle), Rome, 2007,p. 97-251.

8. R. MONIER, Les Institutions financières du comté de Flandre du XIe siècle à 1384, Paris, 1948 ; A. E. Verhulst et M. Gysseling, Le Compte général de 1187 connu sous le nom de "Gros brief » et les institutions financières du comté de Flandre au XII siècle, Bruxelles, 1962.

9. J.-P. GENET, « Conclusion [Les Chambres des comptes en France aux XIV et $\mathrm{XV}$ siècles] »...

10. G. SIVÉRY, Les Capétiens et l'argent au siècle de saint Louis : essai sur l'administration et les finances royales au XIII siècle, Villeneuve d'Ascq, 1995, p. 44. 
Une nouvelle génération de comptes émerge progressivement. La « révolution de l'écrit », le recours aux nombreuses innovations souvent empruntées à d'autres pratiques documentaires (registre et colonnes claires d'origine littéraire, marques graphiques, numérotation), la diversification des formes de «livres outils » progressent justement entre 1250 et 1350 , souvent avant les Chambres des comptes ${ }^{11}$. La fonction crée l'organe, comme l'avait compris Philippe de Mézières, dénonçant « la multitude des livres et des papiers des comptes du royaume de France, pour laquelle multitude il fault tant d'officiers ${ }^{12}$. L'inflation documentaire et sa complexification expliquent de fait en grande partie le besoin d'un système solide de contrôle. L'innovation comptable rend nécessaire le changement institutionnel : le développement de comptabilités et d'archives plus complexes gérées par des receveurs professionnels exige ainsi la mise en place d'une Chambre des comptes à Paris ${ }^{13}$. Le passage du rouleau trimestriel au registre annuel en Artois est lié à la professionnalisation des comptables, devenus des receveurs, capables de rédiger eux-mêmes un compte assez lourd ; il s'explique aussi par l'adoption de nouvelles pratiques financières, par l'introduction des décharges dans les années 1340, qui permettent de décharger la caisse centrale du prince sur les caisses locales au fur et à mesure de la gestion, de sorte que l'on n'ait pas à attendre la reddition du compte local pour en prélever le surplus. La reddition trisannuelle perd dès lors de son intérêt, mais le système exige désormais des contrôles croisés des documents, qui doivent être comparés et consultés après coup ${ }^{14}$. En Bourgogne, on bascule selon une même chronologie vers les registres, et ce bien avant la mise en place d'une Chambre des comptes ${ }^{15}$.

La fondation de ces dernières n'explique en effet pas tout. Il faut rappeler le rôle de la crise économique des années 1330-1380, qui incite ceux qui vivent de leurs rentes à un gros effort de mise à jour documentaire ${ }^{16}$. En Hainaut, l'absence de Chambre des comptes n'empêche

11. P. BERTRAND, Les Écritures ordinaires : sociologie d'un temps de révolution documentaire (entre royaume de France et empire, 1250-1350), Paris, 2015. p. 151-292.

12. PhilipPe DE MÉZIÈres, Le Songe du vieil Pèlerin, cité par P. Contamine, «Introduction », dans P. Contamine et O. Mattéoni éd., La France des principautés..., p. XXXI-XXXVII.

13. É. LALOU, « La chambre des comptes de Paris : sa mise en place et son fonctionnement fin $\mathrm{XIII}^{\mathrm{e}}-\mathrm{XIV}^{\mathrm{e}}$ siècle », dans P. CONTAMINE et O. MATTÉONI éd., La France des principautés..., p. 3-16.

14. J.-B. SANTAMARIA, « Ruptures politiques et mutations comptables au bailliage

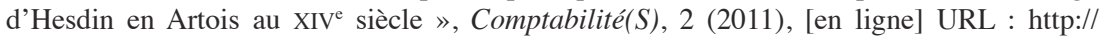
journals.openedition.org/comptabilites/423, consulté le 14 janvier 2019.

15. R. H. BAUTIER, J. SORNAY et F. MURET, Les Sources de l'histoire économique et sociale du Moyen Âge, Paris, 2001, p. 182.

16. G. BoIs, « Noblesse et crise des revenus seigneuriaux en France aux XIV et $\mathrm{XV}^{\mathrm{e}}$ siècles », dans La Noblesse au Moyen Âge. XI ${ }^{e}-X V^{e}$ siècles, Paris, 1976, p. 219-233. 
pas, en 1362, la diffusion au niveau local d'un nouveau modèle de comptes élaboré pour les comptabilités centrales et calqué sur les nouveaux usages, attestés notamment en France ${ }^{17}$. Ce sont les individus qui semblent avoir été essentiels lorsque des institutions pérennes n'existent pas encore, en particulier les marchands et Lombards, comme Tommaso Fini en Flandre au début du XIV siècle $^{18}$. Ces étrangers choisis par le prince introduisent des usages inconnus localement. Leur influence n'est cependant pas toujours pérenne ; ils sont parfois expulsés au gré de la conjoncture politique, et leurs savoirs ne sont pas toujours transmissibles ou peuvent s'avérer inutiles à la gestion «publique », comme la comptabilité à partie double ${ }^{19}$. La position des clercs est différente : bien mieux enracinés et capables de transmettre à leurs homologues, ils préfigurent une plus grande stabilité ${ }^{20}$. La prépondérance des chanoines dans le contrôle et la réforme des comptes est patente entre 1347 et 1382 en Artois, où les innovations aboutissent à un type documentaire stable sans institution fixe ${ }^{21}$. Enfin, le vivier bureaucratique créé par l'administration fournit des compétences dont les maîtres des comptes flamands sont un bel exemple, avec des charges fixes préexistant à l'institution et introduisant les usages parisiens, comme leurs homologues bourguignons durant le premier tiers du XIV siècle, capables de transmettre et de stabiliser leurs pratiques au sein du conseil ou d'un groupe plus restreint d'officiers ${ }^{22}$.

\section{Des institutions de rattrapage?}

C'est donc pour généraliser ou gérer ces innovations que les Chambres des comptes sont mises en place, dans un contexte de baisse des ressources ; la Chambre dijonnaise doit ainsi veiller à la « conservacion de tout le

17. G. SIVÉRY, «L'évolution des documents comptables dans l'administration hennuyère de 1287 à 1360 environ », Bulletin de la Commission Royale, 141 (1975), p. 133-235.

18. W. P. Blockmans, "Financiers italiens et flamands aux XIII-XIV siècles », dans B. Dini éd., Aspetti della vita economica medievale, Florence, 1985, p. 192-214.

19. G. SIVÉRY, «L'évolution des documents comptables...» ; Eddy VAN CAUWENBERGHE, Het vorstelijk domein en de overheidsfinanciën in de Nederlanden (15 de en $16^{\text {de }}$ eeuw). Een kwantitatieve analyse van Vlaamse en Brabantse domeinrekeningen, Bruxelles, 1982, p. 36.

20. T. PÉCOUT éd., De l'autel à l'écritoire. Genèse des comptabilités princières en Occident (XII ${ }^{e}$-XIV siècle), Paris, 2017.

21. J.-B. SANTAMARIA, « Les ecclésiastiques et la comptabilité des princes : Bourgogne, Pays-Bas méridionaux, $\mathrm{XIV}^{\mathrm{e}}-\mathrm{XV}^{\mathrm{e}}$ siècles », dans T. PÉCOUT éd., De l'autel à l'écritoire..., p. 69-103.

22. H. JASSEMIN, « Le contrôle financier en Bourgogne sous les derniers ducs capétiens (1274-1353) », Bibliothèque de l'École des chartes, 79 (1918), p. 102-141 ; F. BLOCKMANS, « Le contrôle par le prince des comptes urbains en Flandre et en Brabant au Moyen Âge », dans Finances et comptabilité urbaines du XIII ${ }^{e}$ au XVI siècle, Bruxelles, 1964, p. 287-338. 
domaine $^{23} »$. Assurément, le mirage de l'État innovant contre lequel Marc Boone nous met en garde ne doit pas tromper ${ }^{24}$. Il s'agit plutôt d'un temps de cristallisation des innovations accumulées qui permet leur enracinement, ce qui n'est pas négligeable, et n'exclut pas la création d'autres instruments. Même si elles sont «tard venues », nul doute, comme le souligne Olivier Mattéoni, que " les Chambres des comptes [...] ont été des lieux de l'innovation et de la rationalisation des pratiques administratives ${ }^{25} »$. On l'observe dans les documents comptables eux-mêmes et dans les instruments « paracomptables » développés par les Chambres.

Le modèle des registres comptables princiers homogènes avec ses trois colonnes, ses titres identifiés, ses rubriques thématiques, s'est souvent diffusé avec les Chambres : le Luxembourg les adopte en passant sous le contrôle de la Chambre parisienne en 1388, puis lilloise un demi-siècle plus tard ${ }^{26}$. L'évolution des comptes de la châtellenie de Château-Regnault, avant et après la fondation de cette dernière, est un exemple patent parmi des milliers d'autres :

La systématisation du registre au moment de la création de la Chambre lilloise est évidente ${ }^{27}$; l'Artois connaît le même phénomène vers 1350 avec la mainmise de la Chambre royale. La foliotation doit beaucoup aux Chambres ; en Bourgogne la fondation de la Chambre dijonnaise généralise son usage, pour permettre les corrections de compte à compte par des renvois précis ${ }^{28}$. L'annualisation s'impose à Lille, Dijon, mais aussi à Grenoble ${ }^{29}$. L'adoption de plans thématiques et d'une classification selon la nature de ce qui est compté se systématise et on retrouve partout

23. P. RiAndey, L'Organisation financière de la Bourgogne sous Philippe le Hardi, Dijon, 1908, p. 175-187.

24. M. BOONE, «L'État bourguignon, un État inventeur ou les limites de l'invention », dans W. PARAVICINI éd., La Cour de Bourgogne et l'Europe. Le rayonnement et les limites, Ostfildern, 2013, p. 133-156.

25. O. MAtTÉONI, «L'étude des Chambres des comptes en France à la fin du Moyen Âge : bilan, débats et enjeux », dans D. LE PAGE éd., Contrôler les finances sous l'Ancien Régime, Paris, 2011, p. 63-80.

26. J.-M. YANTE, « Des comptabilités princières luxembourgeoises de la fin du XIV siècle aux comptabilités bourguignonnes en Luxembourg au milieu du $\mathrm{XV}^{\mathrm{e}}$ siècle. Permanences et mutations », Comptabilité(S), 4 (2012), [en ligne] URL : http://journals.openedition.org/ comptabilites/1079, consulté le 14 janvier 2019.

27. J.-B. SAnTAMARIA, La Chambre des comptes de Lille de 1386 à 1419 : essor, organisation et fonctionnement d'une institution princière, Turnhout, 2012, p. 187-188.

28. S. BepoiX, F. COUVEL et M. LEGUIL, « Entre exercice imposé et particularismes locaux. Étude codicologique des comptes de châtellenie des duché et comté de Bourgogne de 1384 à 1450 », Comptabilité(S), 2 (2011), [en ligne] URL : http://journals.openedition.org/ comptabilites/491, consulté le 14 janvier 2019.

29. A. LEMONDE-SANTAMARIA, Le Temps des libertés en Dauphiné : l'intégration d'une principauté à la couronne de France, 1349-1408, Grenoble, 2002, p. 183-192. 


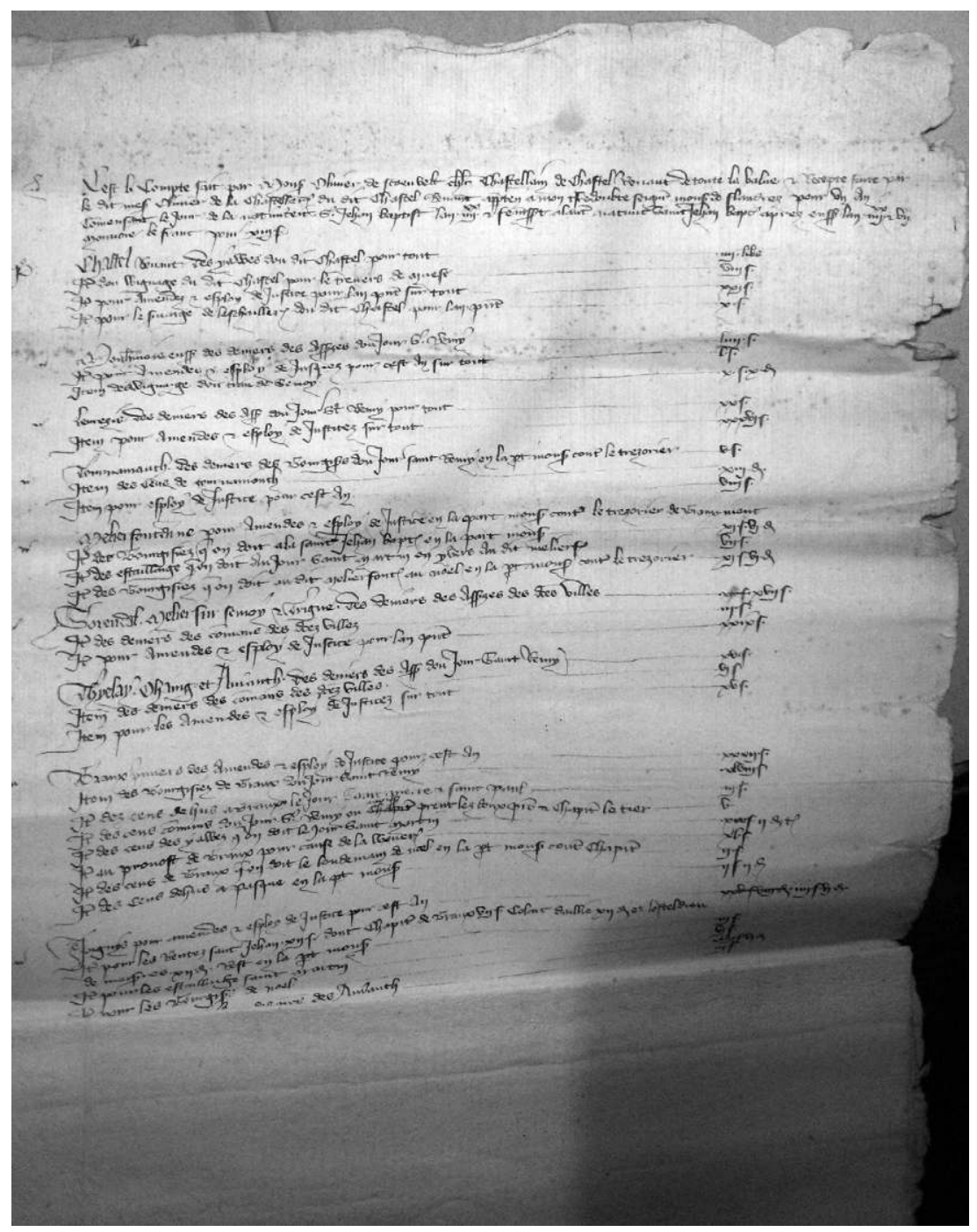

Fig. 1. La comptabilité du comté de Rethel avant la fondation de la Chambre des comptes de Lille : compte de la châtellenie de Château-Regnault en 1380

Source : Lille, AD Nord, B 7348. 


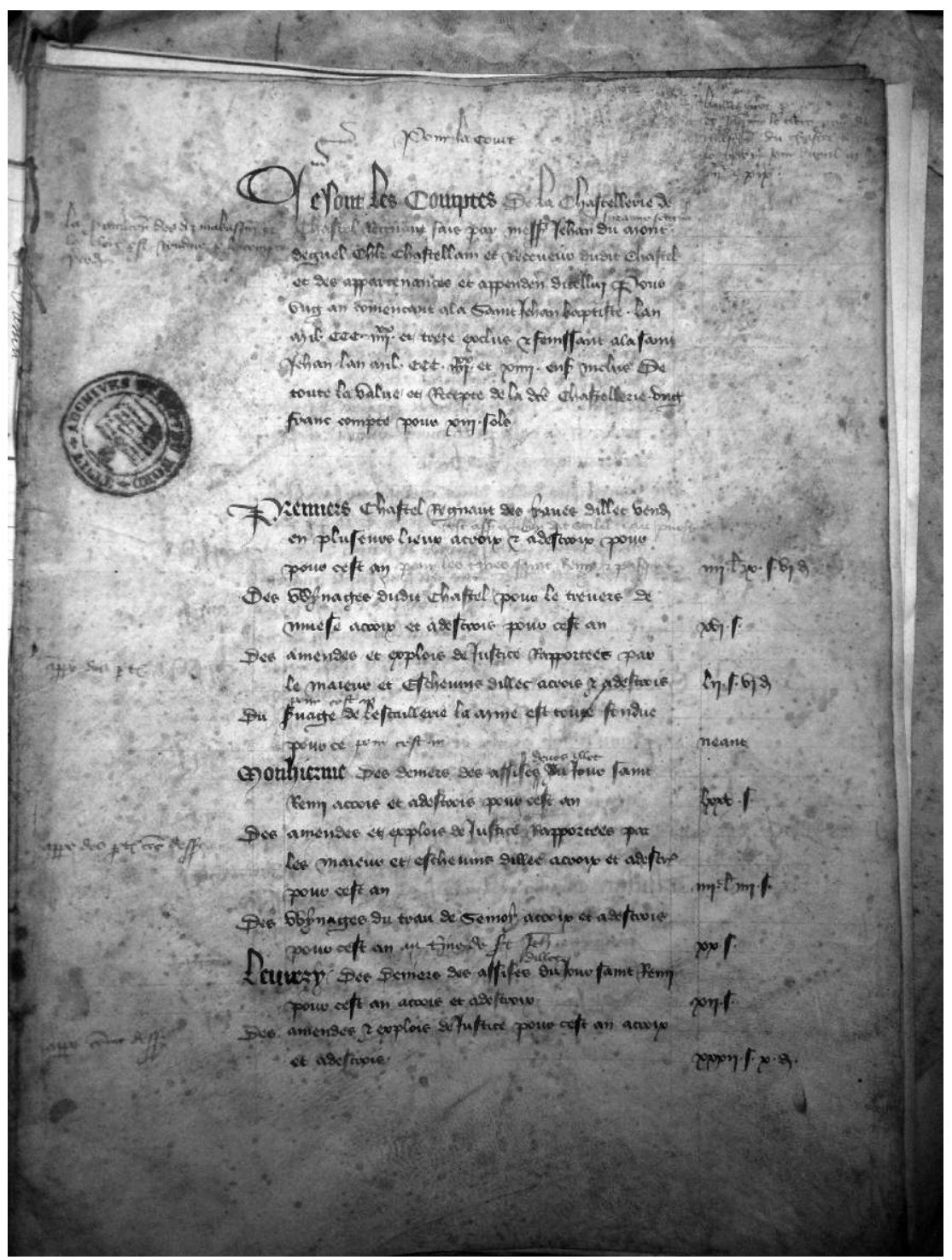

Fig. 2. La comptabilité du comté de Rethel après la fondation de la Chambre des comptes de Lille : compte de la châtellenie de Château-Regnault en 1396 Source : Lille, AD Nord, Cumulus 16148. 
les mêmes marges destinées aux remarques des gens des comptes ${ }^{30}$. Les comptes atteignent d'ailleurs une dimension narrative dans la description des dépenses ${ }^{31}$. Certes, ces innovations avaient pu être introduites par morceaux là où il n'y avait pas de Chambres des comptes, en Artois, en Hainaut, nous l'avons vu. Mais, désormais, elles sont synthétisées pour former un «style » parfois indépassable.

Avec la stabilisation du contrôle et des dépôts, de nouveaux outils fleurissent dans les Chambres, ce que Jean-Paul Boyer a qualifié, pour la chambre aixoise, de "révolution culturelle ${ }^{32}$ ». On retrouve le même processus dans les municipalités italiennes du XIII ${ }^{\mathrm{e}}$ siècle, l'administration anglaise, voire l'université ${ }^{33}$ : des manuels poussent à systématiser les usages en mettant une expérience commune à profit. Ces outils sont en partie imposés par les princes. L'ordonnance du Vivier-en-Brie de 1320 oblige à la tenue d'un journal « pour enregistrer toutes choses qui seront faittes » et impose un inventaire de tous les écrits ${ }^{34}$. Il en est de même à Moulins ${ }^{35}$ comme à $\operatorname{Dijon}^{36}$, où la tenue du « livre des memorialx » est rendue obligatoire en 1386 pour enregistrer les décisions prises par l'institution. La tenue d'un livre des dettes, ou "livre des charges », est également prescrite pour relever tous les impayés glanés à la lecture des comptes ${ }^{37}$. Les Chambres créent des instruments permettant de communiquer entre elles au sein de l'État bourguignon pour croiser les données des chambres de Lille et Dijon, pour s'assurer que les versements d'officier à officier correspondaient, usage qui est étendu à Paris à l'époque où le duc contrôlait la royauté, puis à Bruxelles et La Haye, fondé sur l'échange systématique

30. La comparaison est flagrante : voir le $\mathrm{n}^{\circ} 2$ de la revue Comptabilité(S), Pour une approche codicologique et formelle des documents comptables, [en ligne] URL : https:// journals.openedition.org/comptabilites/364, consulté le 14 janvier 2019.

31. On renverra notamment au $\mathrm{n}^{\circ} 4$ de la revue Comptabilité(S), en particulier l'introduction de P. BECK, « Le vocabulaire et la rhétorique des comptabilités médiévales. Modèles, innovations, formalisation. Propos d'orientation générale », [en ligne], URL : http:// journals.openedition.org/comptabilites/840, consulté le 14 janvier 2019.

32. J.-P. Boyer, « Conclusion. Comptes et histoire totale », dans T. PÉCOUT éd., De l'autel à l'écritoire..., p. 415-440.

33. M. T. Clanchy, From Memory to Written Record : England 1066-1307, Londres, 1979 ; F. MENANT, « Les transformations de l'écrit documentaire entre XII ${ }^{\mathrm{e}}$ et XIII ${ }^{\mathrm{e}}$ siècles », dans N. COQUERY, F. MENANT et F. WEBER éd., Écrire, compter, mesurer. Vers une histoire des rationalités pratiques, Paris, 2006, p. 33-50.

34. É. LALOU, « La chambre des comptes du roi de France », dans P. CONTAMINE et O. MATtÉONI éd., Les Chambres des comptes en France..., p. 1-18.

35. O. MATTÉONI, «Les Chambres des comptes de Moulins, Montbrison et Villefrancheen-Beaujolais à la fin du Moyen Âge », dans P. CONTAMINE et O. MATTÉONI éd., Les Chambres des comptes en France..., p. 43-89.

36. B. SCHNERB, « L'activité de la Chambre des comptes de Dijon entre 1386 et 1404 », dans P. CONTAMINE et O. MATTÉONI éd., La France des principautés..., p. 55-65.

37. L. P. GACHARD éd., Inventaire des Archives des Chambres des Comptes, Bruxelles, 1837-1865, vol. 1, p. 74. 
de données sous forme de cahiers. Émerge ainsi une forme d'intertextualité reliant des institutions distantes de $450 \mathrm{~km}^{38}$.

Ce savoir institutionnel est formalisé dans des ouvrages condensant diverses connaissances pratiques, que l'on peut qualifier de manuels, conservés en plusieurs exemplaires. Celui de la Chambre de Lille, qui date du milieu du $X^{e}$ siècle, mêle science du comput, compilation de serments d'officiers, valeurs des marcs d'or et d'argent et de très riches tableaux permettant d'effectuer des divisions par 365, mais aussi par $4,25,40,100$ et 1000, résultats de calculs besogneusement accomplis à l'abaque et désormais disponibles ${ }^{39}$. Paradoxalement, de telles innovations documentaires ne poussent pas nécessairement les officiers à une maîtrise des méthodes mathématiques, puisqu'il suffit désormais de se conformer à ces tables de manière routinière. Un manuscrit témoigne de préoccupations antérieures à la cour de France, dont les données concernent surtout les années $1300-1335^{40}$. Il n'est pas étonnant que la Chambre des comptes du roi, plus ancienne, ait également eu un manuel des greffiers de la Chambre et un protocole de la Chambre, certes perdu, rédigé par Jean le Bègue ${ }^{41}$. L'instabilité monétaire des $\mathrm{XIV}^{\mathrm{e}}-\mathrm{XV}^{\mathrm{e}}$ siècles a suscité ce type d'outils, que l'on retrouve dans les « livres des changeurs », souvent antérieurs à ceux des Chambres, et qui présentent des similarités ${ }^{42}$, notamment la compilation de tableaux de conversion et de calculs complexes transmis d'une génération à l'autre ${ }^{43}$. Or, certains changeurs devinrent maîtres des comptes à Lille, comme Lotart Frémault ${ }^{44}$, ce dernier étant en fonction au moment où le manuel lillois fut rédigé. La chronologie semble ici indiquer un transfert vers les institutions de savoirs «privés » une fois ces institutions établies. Cette capacité à implanter et transmettre l'innovation explique en partie le succès des institutions.

38. J.-B. SANTAMARIA, « Le contrôle de la recette générale de toutes les finances des ducs de Bourgogne par les Chambres des comptes de Dijon et de Lille : l'État bourguignon entre centralisation et bipolarité (1384-1419) », dans A. JAMME éd., Contrôler les comptes au Moyen Âge. Rites, techniques, portées, XIII $-X V^{e}$ siècle, à paraître.

39. Bruxelles, Archives générales du royaume (désormais abrégé : AGR), CC 1324.

40. Paris, BnF, fr. 11709, f. 160-172.

41. D. PrÉvost, Le Personnel de la Chambre des comptes de Paris de 1320 à 1418, thèse de doctorat, sous la direction de C. Gauvard, Université Panthéon-Sorbonne, 2000, p. 431 .

42. M. BOMPAIRE, «Compétences et pratiques de calcul dans les livres de changeurs français (XIV"e-XVI ${ }^{\mathrm{e}}$ siècle) », dans N. COQUERY, F. MENANT, F. WEBER éd., Écrire, compter, mesurer..., p. 143-162.

43. Y. CoATIVY, « Des catégories aux pratiques. L'outillage mental des changeurs, en France, à la fin du Moyen Âge », Annales des Mines-Réalités industrielles, 2009/1, p. 10-18.

44. J.-B. SANTAMARIA, La Chambre des comptes de Lille..., p. 148 et 160. 


\section{Enraciner l'innovation}

Les Chambres sont certes actives dans la propagation d'innovations, mais leur action s'inscrit dans d'autres logiques : conservation, homologation, uniformisation, authentification l'emportent. Elles sont surtout porteuses d'une autorité princière ; or, la relation entre innovation et autorité est chose complexe.

La circulation des usages d'une Chambre à l'autre dépend en partie d'enjeux politiques impliquant les rapports hiérarchiques entre princes et les liens généalogiques. L'honneur exige qu'on ne copie que les plus glorieuses institutions. À ce titre, l'imitatio regis est un puissant facteur de rationalisation et d'homogénéisation ${ }^{45}$. Le XIV ${ }^{\mathrm{e}}$ siècle voit se diffuser le principe d'imitation des pratiques parisiennes au gré des réformations et fondations de Chambres à Dijon ou Grenoble, puis de réplications des usages dijonnais vers Lille puis Bruxelles. L'existence d'une tradition royale antérieure est un argument fréquemment employé, et permet d'harmoniser les comptes afin de favoriser des comparaisons et de croiser les données : c'est le cas entre Paris et Grenoble pour la royauté ${ }^{46}$, et entre Paris, Lille et Dijon pour les ducs de Bourgogne qui disposent d'espions à la chambre du roi ${ }^{47}$. Nulle honte donc à introduire des usages royaux : à Dijon en 1403, l'audition des comptes est modifiée car « en dit que ainsi est il acoustumé de fere en la chambre des comptes du roy ${ }^{48} »$. Par imitation, la diffusion des Chambres des comptes a déteint sur de grandes maisons : le duc de Bedford a sa Chambre de Mantes ${ }^{49}$, les La Trémoille ou Gilbert de Chabannes affirmaient avoir leur « chambre des comptes ${ }^{50} »$. Mais l'imitation n'empêche pas les pratiques locales ; à Lille, une innovation propre aux usages flamands et artésiens, l'usage de notes marginales en français, a été conservée, à la différence de l'usage parisien du latin ${ }^{51}$.

45. O. MATTÉOnI, " "Imitatio regis". Les institutions financières du comté de Forez et de la seigneurie de Bourbon au début du XIV siècle. De l'influence monarchique et du rôle des hommes : étude comparée », dans P. Contamine, J. Kerhervé et A. Rigaudière éd., Monnaie, fiscalité et finances au temps de Philippe le Bel, Paris, 2007, p. 51-104.

46. A. Lemonde-Santamaria, Le Temps des libertés en Dauphiné..., p. 187.

47. J.-B. SANTAMARIA, « Le contrôle de la recette générale... »; P. CONTAMine, «L'audition des comptes seigneuriaux : l'exemple de la maison de La Trémouille », dans P. CONTAMine et O. MATtÉOni éd., La France des principautés..., p. 259-266.

Voir notamment Bruxelles, AGR, CC 2439.

48. B. SCHNERB, «L'activité de la Chambre des comptes de Dijon... », p. 62.

49. A. CURRY, «L'administration financière de la Normandie anglaise », dans P. CONTAMine et O. MATTÉONi éd., La France des principautés..., p. 83-103.

50. P. CONTAMINE, «L'audition des comptes seigneuriaux : l'exemple de la maison de La Trémouille », dans P. Contamine et O. MATtÉOnI éd., La France des principautés..., p. 259-266.

51. Voir notamment Bruxelles, AGR, CC 2439. 
Dès lors, les comptables sont soumis aux exigences des chambres : l'homogénéisation est sensible pour les domaines intégrés tardivement ou provisoirement. C'est le cas du comté de Saint-Pol sous Jean sans Peur : le receveur fournit à la Chambre lilloise un cahier de papier elliptique, que l'institution fait refondre en exigeant séparation des recettes et dépenses, parchemin, alignement sur le format des comptes ducaux ${ }^{52}$. Reste que le retour dans le giron seigneurial entraîne parfois la fin de ces pratiques, comme le montre la série comptable de la seigneurie de Vitteaux au duché de Bourgogne ; les Chalon s'avèrent peu sensibles aux usages dijonnais ${ }^{53}$.

Les villes doivent aussi souvent adopter les usages princiers, du moins quand leurs comptes n'ont pas déjà connu toutes ces évolutions, car elles ne sont pas forcément « en retard ». À Bruges, la mise en forme est précocement basée sur les registres et suit sa propre voie en adoptant le flamand dans les années 1320 , et en conservant l'usage du papier ${ }^{54}$. À Grenoble, le développement des comptabilités princière et consulaire s'est fait en parallèle, sans lien direct ${ }^{55}$. Mais souvent l'autorité princière impose des réformes à la documentation : cette uniformisation est sensible à partir de 1386 dans les comptes du Franc de Bruges. L'imitation est telle qu'en proclamant leur autonomie en 1488, les villes autonomes de Flandre créent une Chambre des comptes à Gand ${ }^{56}$. On ne pouvait décidément plus s'en passer... Les Chambres des comptes pèsent ainsi sur les comptabilités urbaines de Rennes ${ }^{57}$ ou de Lille ${ }^{58}$. Semblable processus se retrouve chez les princes dits de Savoie-Achaïe en Piémont, où les communautés locales (hôpitaux, communes) s'inspirent souvent des techniques comptables et des types textuels princiers, jugés à la fois efficaces et prestigieux ${ }^{59}$. Dans l'affaire, la dimension hiérarchique est importante, et le prince en tire

52. J. B. SANTAMARIA, La Chambre des comptes de Lille ..., p. 192.

53. Je remercie Matthieu Leguil qui doit publier une communication à ce sujet. Sur Vitteaux et les comptes bourguignons, voir M. LEGUIL, « Le châtelain, le compte et le clerc. Les acteurs de la reddition des comptes de châtellenie en Bourgogne aux XIV et XV $\mathrm{XV}^{\mathrm{e}}$ siècles, d'après l'exemple du bailliage d'Auxois », Comptabilité(S), 7 (2015), [en ligne] URL : http:// journals.openedition.org/comptabilites/1740, consulté le 14 janvier 2019.

54. A. VANDEWALLE, « De oudste stadsrekeningen van Brugge. Bij een nieuwe editie », Handelingen Genootschap «Société d'Émulation » Brugge, 133 (1996), p. 139-143.

55. A. LEMONDE, « Le premier banc des comptes delphinal. Composition, influences et pratiques (1307-1340) », dans T. Pécout éd., De l'autel à l'écritoire..., p. 249-268.

56. M. BoONE, «L'influence des pratiques et du savoir-faire "étatiques" dans les comptes des villes flamandes et des principautés des anciens Pays-Bas aux XIV - XV ${ }^{\mathrm{e}}$ siècles », dans P. BECK et O. MATTÉONI éd., Classer, dire, compter..., p. 199-215.

57. J. KERHERVÉ, L'État breton..., t. 1, p. 403.

58. D. Clauzel, « Lille : un laboratoire d'expérimentation pour la Chambre des Comptes ? », dans Liber Amicorum Claude Lannette, Lille, 2001, p. 37-48.

59. P. Buffo, «Gérer la diversité. Les comptables des Savoie-Achaïe face aux comptabilités urbaines et ecclésiastiques », dans T. PÉCOUT éd., De l'autel à l'écritoire..., p. 393-414. 
avantage, car les comptes urbains peuvent aisément être croisés avec les comptes princiers, ce qui est fort utile pour la perception de droits comme les assises.

La logique inverse n'est pas forcément vraie. Les villes sont certes des « lieux d'innovation et de création institutionnelles en matière de gestion fiscale et financière ${ }^{60} »$, mais si les passerelles existent avec les princes, elles ne vont pas de soi, contrairement à une doxa présentant l'essor de l'État comme un transfert de compétence depuis les «bourgeois calculateurs $^{61} \gg$. On ne niera nullement l'importance du recrutement bourgeois dans ces institutions ${ }^{62}$ et parmi les comptables eux-mêmes, notamment en Bourgogne ducale ${ }^{63}$, mais cela ne signifie pas qu'on imite les usages urbains. La diffusion des pratiques nouvelles ne peut se comprendre par une simple contamination de proche en proche, car elle peut répondre à des logiques politiques, voire d'orgueil ; Robert d'Artois fait ainsi venir un spécialiste des finances d'Italie du Sud pour introduire les registres de comptes en Artois, alors que de grandes villes du Nord comme Bruges l'utilisent déjà dans les années $1283-1284^{64}$. La nature conflictuelle de ses liens avec les villes de Flandre ne l'a sans doute pas incité à aller trouver chez elles les modèles de son administration. L'imitation se fait de haut en bas, d'autant que l'innovation permet aussi d'imposer une autorité, parfois avec morgue. On retrouve cette attitude à Dijon et à Lille à l'égard des agents locaux lorsque les Chambres se constituent, avec cette nuance que les gens des comptes sont surtout valorisés en tant que conservateurs du domaine et du pouvoir princier, " gens de prudence et experts », « moult expert oudit fait de comptes », nullement comme inventeurs.

Par ailleurs, les usages ainsi introduits sont destinés à être pérennes, et à produire des séries documentaires relativement constantes et conservées dans des archives stables dont les Chambres des comptes sont la plus belle incarnation. Faute de stabilité ou de conservation, bien des innovations ne

60. D. Menjot et M. SÁnchez Martínez, «Introduction », dans D. Menjot et M. SÁNCHEZ MARTíneZ éd., La Fiscalité des villes au Moyen Âge. 4, Toulouse, 2004, p. 5-8.

61. W. PARAVICINI, «Administrateurs professionnels et princes dilettantes. Remarques sur un problème de sociologie administrative à la fin du Moyen Âge », dans K. F. WERNER et W. PARAVICINI éd., Histoire comparée de l'administration : IVe-XVIII siècles, Munich/Zürich, 1980, p. 168-181 ; W. P. BLOCKMANS, « Princes conquérants et bourgeois calculateurs. Le poids des réseaux urbains dans la formation des états », dans La Ville, la bourgeoisie et la genèse de l'État moderne, Paris, 1988, p. 167-181.

62. J.-B. SANTAMARIA, La Chambre des comptes de Lille ..., p. 119-166 ; J. DUMOLYN, Staatsvorming en vorstelijke ambtenaren in het graafschap Vlaanderen (1419-1477), Anvers, 2003.

63. Ils représentent plus de la moitié des comptables ducaux en Auxois : M. LEGUIL, « Le châtelain, le compte et le clerc...».

64. B. Delmaire, Le Compte général du receveur d'Artois pour 1303-1304, Bruxelles, 1977, p. XLVIII. 
sont pas durables, ou du moins ne sont pas conservées. Un contre-exemple nous est donné par un autre type de document dérivé des comptes, l' " estat par provision », ce qui se rapproche le plus du budget. Ces documents sont rarissimes et souvent tardifs : on les fait souvent remonter au milieu du $X^{\mathrm{e}}$ siècle $^{65}$. Pourtant, cette impression est liée à leurs conditions de rédaction : rédigés par des conseillers, et non par une institution permanente, pour des circonstances précises, ils sont destinés à être détruits car sans intérêt archivistique et surtout dangereux s'ils tombaient en de mauvaises mains. D'où la croyance, chez un auteur comme Jean Rauzier, selon laquelle la gestion princière aurait été caractérisée par « l'absence presque totale de prévisions ${ }^{66}{ }^{»}$. Or, on dispose de prévisions dans les années 1330-1340 pour la cour de France ${ }^{67}$ et la Flandre ${ }^{68}$. De tels documents sont cependant destinés à disparaître. On sait que Louis XI faisait détruire ce type de rapports appelés « grand secraiz de finance », qui lui donnaient bien des frayeurs ${ }^{69}$. Au total, c'est cette politique de destruction, ainsi que l'absence de « conseil des finances » stable, qui a laissé croire que ces projections furent une innovation tardive.

\section{Combattre l'innovation?}

Les Chambres n'ont pas vocation à innover. Une fois passée la phase d'installation, le rapport à l'innovation s'avère même compliqué. Peu désireuses de passer pour innovantes, elles ont tendance à faire passer des formes nouvelles pour des usages anciens. On continue d'appeler en 1341 le compte de la saunerie de Salins « rolle », alors qu'il s'agit d'un registre $^{70}$. La recette générale des finances bourguignonne disposait d'un « contrerolle », qui n'est pas un rouleau mais un cahier. La comptabilité est pleine de mots anciens pour des réalités nouvelles. On désigne les usuriers, lombards ou non, comme «marchands » ou « changeurs »; on qualifie de

65. M. ARnOuld, «Une estimation des revenus et des dépenses de Philippe le Bon en 1445. Recherche sur l'histoire des finances publiques en Belgique », Acta Historica Bruxellensia, 3 (1974), p. 131-239.

66. J. RAUZIER, Finances et gestion d'une principauté : le duché de Bourgogne de Philippe le Hardi, 1364-1384, Paris, 1996, p. 710.

67. J. FAVIER éd., Finance et fiscalité au bas Moyen Âge, Paris, 1971, p. 295-304.

68. P. THOMAS, «Une source nouvelle pour l'histoire administrative de la Flandre : le registre de Guillaume d'Auxonne, chancelier de Louis de Nevers, comte de Flandre », Revue du Nord, 10 (1924), p. 5-38.

69. J.-F. LASSALMONIE, La Boîte à l'enchanteur : politique financière de Louis XI, Paris, 2002, p. 6 et 247.

70. S. Le StRat-Lelong, Le Comté de Bourgogne d'Eudes IV à Philippe de Rouvres (1330-1361) : une principauté en devenir, thèse de doctorat, sous la direction de M. Bubenicek, Université de Besançon, 2015, p. 164. 
« dons» ou « courtoisies» du prince des versements correspondant à des taux d'intérêt précis ${ }^{71}$.

Enfin, certaines innovations documentaires comptables semblent avoir suscité l'opposition des gens des comptes. Si le papier a été introduit dans la comptabilité du comté de Bourgogne en 1329, il n'en est pas question dans le duché qui voit émerger plus tôt une Chambre des comptes ${ }^{72}$. Quant aux chiffres arabes, rappelons qu'ils furent introduits en 1766 à la Chambre des comptes du roi. Au point que l'Encyclopédie évoque, sans d'ailleurs la critiquer, l'existence d'un «chiffre françois » qui est le « chiffre de finance » utilisé à la Chambre des comptes ${ }^{73}$. Combat d'arrière-garde ? Alain Schärlig a montré que la très lente diffusion des chiffres arabes, phénomène assez généralisé, s'explique en vérité par l'efficacité pratique de l'abaque : dans la mesure où celle-ci permet de réaliser aisément des opérations à partir de chiffres romains, elle retarde la nécessité d'adopter le calcul et les chiffres arabes. La fiabilité du calcul avec des jetons pour les comptes princiers n'est d'ailleurs plus à démontrer ${ }^{74}$. L'outillage mental d'un changeur permet en outre d'affronter des problèmes très complexes avec ces méthodes, adaptées au système livre/sou/denier, non décimal, et donc peu compatible avec le calcul par chiffres arabes ${ }^{75}$. La méthode de l'abaque correspond mieux au fonctionnement d'une institution collégiale, le calcul étant effectué sous le regard des autres membres et même du comptable, assurant une relative transparence, voire effectué en parallèle par plusieurs personnes, ce dont témoigne l'iconographie. Enfin, les Chambres sont des cours de justice soumises à une procédure, un stylus curiae, gage de validité. Les chiffres arabes sont à ce titre réputés plus faciles à falsifier. Cela n'empêche pas les gens des comptes de s'y intéresser dans leurs manuels ${ }^{76}$; dès 1474 , en Flandre, des pièces puis, au $\mathrm{XVI}^{\mathrm{e}}$ siècle, des jetons introduisirent ces chiffres ${ }^{77}$. Par ailleurs, les réticences sont plus larges : au début de l'époque moderne, on continue d'exiger la maîtrise de l'abaque par les changeurs. C'est à partir du XVI siècle, mais très lentement, que l'usage se répand ; le $\mathrm{XVII}^{\mathrm{e}}$ siècle voit encore les traités sur l'abaque largement diffusés chez les marchands comme chez les particuliers ${ }^{78}$. À l'inverse, les Chambres des comptes connaissent un intérêt réel pour le savoir mathématique, nullement

71. J.-B.SANTAMARIA, Le Secret du prince. Gouverner par le secret, France-Bourgogne, $X I I I^{e}-X V^{e}$ siècle, Ceyzérieu, 2018, p. 242.

72. M. LEGUIL, «"Faire et ordonner ses comptes"....».

73. Article «Finances », Encyclopédie méthodique, Paris/Liège, t. I, 1784, p. 294.

74. Certains comptes ne comportent aucune erreur sur 100 folios :

75. M. BOMPAIRE, « Compétences... ».

76. Ibid.

77. J. RouYer et E. F. F. HuCHER, Histoire du jeton au Moyen Âge. Première partie, Paris, 1858.

78. M. BOMPAIRE, « Compétences... ». 
réservé aux marchands. Dès le XIV ${ }^{\mathrm{e}}$ siècle, des questions d'avaluement des monnaies ${ }^{79}$, qui requièrent des calculs complexes, sont traitées à la Chambre des comptes, et des mathématiciens de haut niveau s'y trouvent à la fin du $\mathrm{XV}^{\mathrm{e}}$ siècle comme Jean Adam, auteur du Traité en arismectique pour la pratique par gectouers en $1475^{80}$.

Reste qu'il y eut sans doute un « effet institution ». C'est souvent à ses débuts qu'une Chambre des comptes peut avoir une action « innovante »; les grands règlements pour Paris en 1320, Lille et Dijon en 1386, établissent de nouveaux usages, mais on peut se demander si à terme ces institutions de "réforme », en devenant permanentes, n'ont pas perdu de cet esprit, comme d'autres institutions avant elles, à l'instar de la chambre des Renenghes établie quelques siècles auparavant en Flandre. Les séries comptables montrent effectivement qu'après les grands changements du XIV ${ }^{\mathrm{e}}$ siècle on arrive à une réelle stabilité, de l'Artois à la Bourgogne, qui favorise la comparaison d'un territoire à l'autre, ou d'une année sur l'autre. À Lille, les gens des comptes s'opposent dès lors avec succès à une tentative de réforme de Philippe le Bon, qui souhaite leur faire tenir un compte séparé des diverses monnaies comme à Dijon ${ }^{81}$. Ceux de Dijon s'ingénient à saboter la réforme de la recette générale de Bourgogne menée par Charles le Téméraire, recommandant avec succès d'agir « sans y faire autre mutacion ne innovacion » en raison des complications engendrées, et faisant d'ailleurs perdurer un découpage des circonscriptions administratives jusqu'à la Révolution ${ }^{82}$. Les institutions ne sont pas destinées à l'innovation permanente, mais à réformer une fois pour toute un système suite à une crise ; leur création relève souvent d'un tour de passe-passe consistant à les faire passer pour de simples continuations de pratiques antérieures. Elles partagent d'ailleurs la vision conservatrice de la société, qui est celle du prince comme des différents corps constitués ${ }^{83}$. Elles se moulent dans la hiérarchie de ces corps, étant reconnues comme des «collèges » ou des « communautés », faisant de leurs chartes de fondation des privilèges qui leur

79. C'est-à-dire d'estimation, d'évaluation des monnaies par rapport aux métaux précieux, aux autres pièces, ou au cours des monnaies de compte.

80. S. LAMASSÉ, «Calcul et marchandises (XIV"-XV siècles) », dans L. MoULINIERBrogi et al. éd., La Juste Mesure. Quantifier, évaluer, mesurer entre Orient et Occident, Saint-Denis, 2005, p. 79-98.

81. J.-B. SANTAMARIA, «Un maître prévaricateur à la Chambre des comptes de Lille sous Philippe le Bon : Roland du Bois », dans W. PARAVICINI et B. SchnerB éd., La Face noire de la splendeur. Crimes, trahisons et scandales à la cour de Bourgogne aux XIVe et $X V^{e}$ siècles, Villeneuve d'Ascq, 2009, p. 421-447.

82. P. CoCKSHAW, « Heurs et malheurs de la recette générale de Bourgogne », Annales de Bourgogne, 41 (1969), p. 247-271.

83. J.-B. SANTAMARIA, «Innover sans l'avouer : les fondations d'institutions de la fin du Moyen Âge », dans É. LECUPPRE-DESJARDIN éd., Innovation, politique, autorité, Villeneuve d'Ascq, à paraître. 
permettent de s'affirmer comme autonomes et protectrices de leurs usages et styles ${ }^{84}$. Encore au $\mathrm{XV}^{\mathrm{e}}$ siècle, la vénalité des charges n'étant pas établie, le prince peut imposer des réformes, lesquelles ne viennent en tout cas pas de l'intérieur. Lorsque Charles VII réinstalle la Chambre à Paris après 1436, il peut mener un vaste plan de réforme et de rationalisation ${ }^{85}$, conduisant à la rédaction d'instruments documentaires nouveaux ${ }^{86}$. De telles réformes se retrouvent encore, à la même époque, à Lille ou à Moulins ${ }^{87}$. À terme cependant, la capacité ou la volonté de réforme du prince semble faiblir. La documentation subit même un mouvement de simplification, qui s'explique par l'affirmation d'une plus grande autonomie des Chambres en raison de la vénalité des offices, mais aussi par un changement dans la fonction du compte et de l'officier comptable. De la Flandre au Genevois, le contenu des comptes perd en richesse à mesure que les offices se trouvent affermés ou vidés de certaines de leurs fonctions, les châtelains perdant des attributs militaires, financiers et judiciaires par exemple ${ }^{88}$. Le mouvement vient aussi des chambres : ces collèges d'experts qui forment en leur sein une bonne part des recrues contribuent à la valorisation d'un savoir pointu, parfois qualifié de jargon ${ }^{89}$, qu'on se transmet en secret entre spécialistes, nourris à la Chambre au «stile de nostre dite Chambre », selon la commission d'un clerc lillois en $1395^{90}$. Cet usage est à rapprocher de la transmission des savoirs dans les métiers ${ }^{91}$, avec lesquels les institutions partagent des problématiques voisines : tiraillées entre réglementation et efficacité, les métiers ne sont pas par nature fermés à l'innovation, comme on le sait pour

84. Ibid.

85. P. CONTAMINE, « Réformer l'État, rationaliser l'administration : à propos du contrôle des finances publiques 1456-1461 », dans Mélanges Jean Favier, Paris, 1999, p. 388-396.

86. P. CONTAMINE, «La mémoire de l'État. Les archives de la Chambre des comptes du roi de France, à Paris, au XV $\mathrm{XV}^{\mathrm{e}}$ siècle », dans Des pouvoirs en France, 1300-1500, Paris, 1992, p. 237-250.

87. G. Castelnuovo, «Service de l'État et identité sociale », Revue historique, 618 (2001), p. 489-510 ; O. Mattéoni, «L'étude des Chambres des comptes... », p. 69.

88. L. PERRILlat, « Le contrôle des comptes et l'enregistrement à la Chambre des comptes de Genevois », dans D. LE PAGE éd., Contrôler les finances sous l'Ancien Régime : regards d'aujourd'hui sur les Chambres des comptes, Vincennes, 2011, p. 259-275.

89. Rabelais compare les notes marginales des gens des comptes aux hiéroglyphes... O. MAtTÉONI, «Vérifier, corriger, juger. Les Chambres des comptes et le contrôle des officiers en France à la fin du Moyen Âge », Revue historique, 641 (2007), p. 31-69.

90. Lille, AD Nord, B 1598, f. 28.

91. P. BRAUnSTEIN, « Savoir et savoir-faire : les transferts techniques », dans P. BECK éd., L'Innovation technique au Moyen Âge, Paris, 1998, p. 303-311 ; É. ANHEIM et F. MENANT, « Mobilité sociale et instruction : clercs et laïcs du milieu du XIII ${ }^{\mathrm{e}}$ au milieu du XIV ${ }^{\mathrm{e}}$ siècle », dans S. CARocci éd., La Mobilità sociale nel Medioevo, Rome, 2010, p. 341-379 ; J.B. SANTAMARIA, «"Expers et cognoissans en fait de comptes"...». 
d'autres milieux comme celui de la métallurgie, mais s'avèrent méfiants vis-à-vis de tout procédé nouveau pouvant discréditer leur compétence ${ }^{92}$.

Nombre de changements dans les usages ont rendu nécessaire la mise en place d'un contrôle par un collège d'experts à plein temps, disposant de ressources et d'archives permettant la confrontation des documents, et capables de générer leurs propres outils documentaires «paracomptables ». Une fois en place, c'est surtout dans la diffusion, la systématisation de toutes les pratiques émergeant au XIII ${ }^{\mathrm{e}}$ siècle, que les Chambres sont durablement efficaces, enracinant l'innovation comptable au point qu'innovations documentaires et institutionnelles sont indiscutablement mêlées. La plus influente, la Chambre des comptes du roi, joue certainement un rôle essentiel dans l'élaboration d'une synthèse de ces procédés en un modèle comptable. Dans le détail, les nuances locales sont réelles, les pratiques locales sont le reflet de la diversité des contraintes imposées aux organes administratifs : idéal conservateur, pragmatisme, promotion d'une culture savante, mais aussi volonté de réforme, et progressivement revendication d'une identité institutionnelle locale. Le tout est également soumis à divers équilibres à l'intérieur de l'institution (statut des officiers, rôle de la collégialité, nature de son recrutement), à l'intérieur de l'État (concurrence d'autres institutions, rapport au prince), aux relations avec d'autres pouvoirs (villes, établissements religieux), enfin au fonctionnement global des finances. Les comptes eux-mêmes sont à la croisée de plusieurs usages entre lesquels il faut arbitrer : enregistrer l'état du domaine, garder la mémoire de l'État, fournir des informations prospectives, vérifier la conformité juridique des opérations comptables, comparer les comptes entre eux. L'introduction d'innovations documentaires répond aussi pour partie à l'évolution dans l'équilibre entre ces fonctions.

L'innovation n'est donc pas à porter au seul crédit des institutions, loin de là. Les changements liés à des individus sont à prendre en compte : le bouleversement de la comptabilité de la ville de Mons en 1345 est le fait d'un clerc de la ville, en dehors de toute Chambre ; la pérennité de tels changements est toutefois moins garantie en l'absence d'institution. L'effet d'une structure est de systématiser, de précipiter l'adoption d'usages parfois longuement mûris. Les institutions transforment les innovations en standards, parfois indépassables, et ce d'autant plus que c'est par son style que l'institution perdure et existe en tant que structure collective, fondant son statut non sur des rapports interpersonnels mais des règles abstraites. C'est

92. J.-F. BELHOSTE, « Les hommes du fer : réflexions sur l'émergence et la diffusion de l'innovation (XIV $-X \mathrm{XII}^{\mathrm{e}}$ siècle) », dans M. ARNOUX et P. MONNET éd., Le Technicien dans la cité en Europe occidentale, 1250-1650, Rome, 2004, p. 271-289 ; C. VERNA et L. HILAIREPÉREZ, «Dissemination of Technical Knowledge in the Middle Ages and the Early Modern Era : New Approaches and Methodological Issues », Technology and Culture, 47 (2006), p. 536-565. 
pourquoi l'institutionnalisation contribue à figer la forme documentaire. Si à terme l' " effet institution » peut entraîner un certain conservatisme, c'est enfin en raison de l'adéquation de la forme à sa fonction : le chiffre romain fait parfaitement l'affaire, et on peut se demander si le changement introduit au XVIII ${ }^{\mathrm{e}}$ siècle n'était pas une concession au goût du jour et à l'émergence d'un intérêt nouveau pour l'innovation en elle-même, plutôt qu'une réforme utile.

Jean-Baptiste Santamaria - Université de Lille, Laboratoire IRHIS (UMR 8529) 
Comptabilité publique et innovation à la fin du Moyen Âge : les institutions princières et l'émergence d'une culture numérique dans un ordre conservateur

Les modifications enregistrées par la documentation en matière de techniques comptables entretiennent des rapports complexes avec un autre phénomène propre à la fin du Moyen Âge : le développement d'institutions stables et normées chargées du contrôle des comptes. Si une première génération d'institutions accompagne la mise en place de toute une série de techniques entre $\mathrm{XI}^{\mathrm{e}}$ et $\mathrm{XIII}$ siècles, ces corps deviennent progressivement inadaptés aux changements rapides des modes de gestion et aux innovations documentaires apparues entre XIII et XIV ${ }^{e}$ siècles. C'est pour répondre à ces nouvelles exigences, parfois promues par la base de l'administration, que les Chambres des comptes se mettent en place au cours du XIVe siècle, parfois avec un peu de retard sur les changements documentaires, mais se faisant immédiatement les promotrices zélées des techniques nouvelles. Une fois installées dans le paysage, ces institutions inventent moins qu'elles n'acclimatent et installent durablement toute une série d'innovations antérieures, dont la circulation répond cependant aussi à des enjeux de préséance et d'honneur. Après cette phase de " réformation ", durant laquelle sont durablement établies les bonnes pratiques, un " effet institution " semble jouer : les Chambres défendent farouchement leur " style " qui devient un élément de leur autonomie ou de leur identité, qui garantit la validité de leurs actions, et les princes peuvent avoir du mal à les inciter à introduire de nouveaux usages.

Chambre des comptes, comptabilité, innovation, institutions, princes.

Public accounting and innovation in the Late Middle Ages: Princely institutions and the growth of numerical culture in a conservative order

The transformations of accounting techniques in the Late Middle Ages have a complex relationship with another phenomenon: the development of stable and regulated institutions responsible for auditing accounts. Although a first generation of institutions accompanied the implementation of a series of techniques between the eleventh and thirteenth centuries, these bodies gradually became unsuited to the rapid changes in management methods and to the documentary innovations that emerged in the thirteenth and fourteenth centuries. It was in response to these new demands, which sometimes stemmed from the administration, that the "Chambres des comptes" emerged during the course of the fourteenth century. Despite initially lagging behind when it came to the documentary changes, they soon became ardent promoters of these new techniques. Once fully established, these institutions did not so much invent as acclimatize, and they implemented a series of past innovations. After this phase of "reformation," during which long-lasting rules of good practice were put into place, an "institution effect" came into play: the "Chambres des comptes" stoutly defended their "style," which became an element of their autonomy and their identity and served to guarantee the validity of their actions. This meant that princes struggled to impose new uses upon them.

Accounting, Chambre des comptes, innovation, institutions, princes. 
